\title{
ESTIMATIVAS DE PARÂMETROS DE CURVAS DE LACTAÇÃO DEBOVINOS
}

\author{
ESTIMATES OF LACTATION CURVE PARAMETERS OF CATTLE \\ Cruz, G.R.B. ${ }^{1}$, M.N. Ribeiro ${ }^{2}$ e E.C. Pimenta Filho ${ }^{3}$
}

${ }^{1}$ Rua Mons. Pedro Anísio B. Dantas, 07.Centro, 58220-000.Bananeiras-PB. Brasil.georgebeltrao@bol.com.br ${ }^{2}$ DZ/UFRPE. Recife-PE.Brasil.mnribeiro@uol.com.br ${ }_{3}^{3}$ ZZ/UFPB/CCA. Areia-PB. Brasil. edgard@cca.ufpb.br

\section{Palavras chave adicionais}

Curvas de lactação. Funções matemáticas. Guzerá. Sindi.

\section{RESUMO}

O objetivo deste trabalho foi comparar algumas funções matemáticas citadas na literatura, identificar a que melhor descreve a produção de leite e estimar alguns componentes da curva de lactação de vacas Guzerá e Sindi criadas na região semiárida do estado da Paraíba. Foram utilizadas 840 lactações (467-Guzerá; 373-Sindi) de animais pertencentes à Fazenda Carnaúba, município de Taperoá, Paraíba. O controle leiteiro foi feito em intervalos de 35 dias, no período de 1987 a 2004. As lactações utilizadas continham no mínimo cinco e no máximo doze controles. $\mathrm{O}$ ajuste foi feito para a curva média e para lactações individuais, utilizando-se seis funções matemáticas. A função polinomial inversa (PI), linear hiperbólica (LHE), gama incompleta (GI), quadrática logarítmica (QL), linear (L) e quadrática (Q) foram ajustadas usando-se processos iterativos por meio de regressão não-linear. Os critérios utilizados para verificar a qualidade do ajuste para cada função foram: coeficiente de determinação ajustado $\left(R_{a}{ }^{2}\right)$, percentagem de desvios entre as produções totais observadas e estimadas e percentagem de curvas típicas. Os gráficos de distribuição de resíduos foram utilizados apenas para avaliar o ajuste para a curva média. Para a curva média, os valores de $R_{a}{ }^{2}$ foram superiores a 0,93 para todas as funções. Bons ajustes baseados nos $R_{a}^{2}>0,80$ foram obtidos, respectivamente, para $61,4 \%$ e $66,7 \%$ das lactações ajustadas pelas funções PI e GI para vacas Guzerá. Para vacas Sindi, esses valores foram de $57,2 \%$ e $55,0 \%$, indicando boa qualidade

Recibido: 4-1-07. Aceptado: 22-10-08.

\section{AdDitional KeYWORDS}

Lactation curves. Mathematical functions. Guzerá. Sindi.

no ajuste. As funções $\mathrm{Pl} \mathrm{e} \mathrm{Gl} \mathrm{acompanharam} \mathrm{mais}$ de perto as oscilações da produção ao longo da lactação. Para curva média, todas as funções poderiam ser utilizadas, pois os desvios foram muito próximos. Para as lactações individuais, melhores estimativas dos componentes da curva de lactação poderiam ser obtidos pelas funções PIeGI.

\section{SUMMARY}

The objective of this research was to identify the mathematical function that best fits the lactation curve of Guzerá and Sindi cattle. 840 lactations records (467-Guzerá, 373-Sindi) were used from animals raised at Carnauba ranch, in the town of Taperoá, Paraíba. Milk production control were taken every in 35 days, between years 1987 and 2004. Lactation records used contained a minimum of five and a maximum of ten controls. Six mathematical functions were fitted to the mean curve and individual lactations: polynomial inverse (PI), hyperbolic lineal (LH), gamma incomplete (GI), quadratic logarithmic $(Q L)$, lineal $(L)$ and quadratic (Q) functions were fitted using an iteration process. Criteria used to verify fitting quality were: determination coefficient $\left(R_{a}{ }^{2}\right.$ ), percent of deviation between the total observed estimated productions, and percent of typical curves. Residual distribution graph were used only to evaluate fitting of mean curve. $R_{a}^{2}$ for mean curve fitting were always larger than 0.93. Good fits were also obtained

Arch. Zootec. 58 (224): 695-704. 2009. 


\section{CRUZ, RIBEIRO E PIMENTA FILHO}

$\left(R_{a}{ }^{2}>0.80\right)$, in $61.4 \%$ and $66.7 \%$ of the lactations fitted by $\mathrm{PI}$ and $\mathrm{Gl}$ functions, respectively, for Guzerá cattle. For Sindi cattle, these values were $57.2 \%$ and $50.0 \%$. PI and $\mathrm{GI}$, respectively, were closest to the production oscillations throughout lactation, even though estimated deviations between total productions were observed and estimated that were similar to the other functions. All studied functions fitted well means curve data. For individual lactations, however, $\mathrm{PI}$ and GI give better estimates of total milk yields.

\section{INTRODUÇÃO}

A curva de lactação é a representação gráfica da produção de leite no decorrer de uma lactação. O Estudo de curvas de lactação pode contribuir na tomada de decisões de manejo alimentar, reprodutivo, descarte de animais ou até mesmo em programas de seleção. A predição da produção total de leite de uma vaca em lactação facilita a escolha antecipada e precisa de machos através de seus descendentes como também a escolha de fêmeas dento do rebanho (Bianchini Sobrinho, 1984).

Uma curva de lactação típica é composta de uma fase inicial, em que a produção aumenta do parto e se estende até a fase de pico, aproximadamente na oitava semana, caracterizada pela produção máxima observada e por fim, uma fase de declínio continuado até o final da lactação (Cunha Filho, 2002). Nas raças zebuínas e seus cruzamentos, a curva de lactação é decomposta em dois segmentos: produção inicial e taxa de declínio da produção, os quais são influenciados por fatores genéticos e ambientais (Cobuci et al., 2001).

As funções matemáticas usadas para representar curvas de lactação são divididas em três tipos: linear, intrinsecamente linear e não linear (Afifi e Clark, 1984). Estudos com curvas de lactação em bovinos tiveram início em 1923 (Brody et al., 1923). Existem na literatura muitas funções matemáticas utilizadas para descrever a curva de lactação de bovinos. As mais utilizadas são as intrinsecamente lineares.
Nelder (1966) propôs a função polinomial inversa. Este modelo foi linearizado por Bianchini Sobrinho (1984) e seus parâmetros estimados pelo método dos quadrados mínimos. Pineda (1987) e ElFaro(1996) também usaram esse modelo para estimativas de curvas de lactação o qual apresentou bastante flexibilidade no ajuste destas curvas.

A função gama incompleta foi proposta por Wood (1967). Este modelo, intrinsecamente linear, é bastante utilizado em estudos de lactação. Os maiores problemas no ajuste deste modelo são as superestimações de produção tanto no início como no final da lactação (Cobby e Le Du, 1978).

Bianchini Sobrinho (1984) propôs a função linear hiperbólica e a função quadrática logarítmica. Para a função linear hiperbólica estimar curvas típicas e picos de lactação os parâmetros $a_{1}$ e $a_{2}$ do modelo matemático devem ser negativos. A função quadrática logarítmica tem sido testada por alguns autores apresentado bons resultados no ajuste (Pineda, 1987; Ribeiro, 1997; Bianchini Sobrinho, 1988).

A função quadrática apresenta ajuste simples apresentando ponto de máximo quando o coeficiente quadrático é menor que zero. Esta função é de fácil utilização e seus parâmetros têm significado biológico. A função linear, apesar de representar curva de lactação de alguns animais, possui a limitação de não acompanhar as oscilações existentes na produção de leite ao longo da lactação, subestimando e superestimando a produção em vários estágios da lactação.

Alguns parâmetros práticos são calculados a partir do modelo utilizado para estimar a curva de lactação de um rebanho. Estes parâmetros são: tempo de pico, produção no pico e persistência. O pico de produção pode ser definido como a produção máxima em uma lactação. A persistência pode ser definida como a extensão em que o pico de lactação é mantido ou a taxa de declínio após o pico de produção (Bianchini Sobrinho, 1984).

O presente trabalho objetivou comparar 
algumas funções matemáticas citadas na literatura, identificar a que melhor descreve a produção de leite e estimar alguns componentes da curva de lactação de vacas Guzerá e Sindi criadas na região semi-árida do estado da Paraíba.

\section{MATERIALEMÉTODOS}

Utilizou-se dados de 840 controles de produção de leite de bovinos, sendo 467 controles da raça Guzerá e 373 controles da raça Sindi, controladas no período de 1987 a 2004, pertencentes à Fazenda Carnaúba, município de Taperoá, Estado da Paraíba. A Fazenda está localizada na microrregião do Cariri Ocidental, a $7^{\circ} 12^{\prime} 23^{\prime \prime}$ de latitude sul e $36^{\circ} 49^{\prime} 25^{\prime \prime}$ de longitude W.G. a uma altitude de $500 \mathrm{~m}$ (IBGE, 1990). OClima da região é de tendência tropical, com precipitação anual média em torno de $300 \mathrm{~mm}$. É caracterizado por apresentar uma curta estação chuvosa e estação seca prolongada, maior que 8 meses, e umidade relativa do ar em torno de $65 \%$.

O controle leiteiro é realizado a cada 35 dias, em duas ordenhas diárias, com intervalo de 12 horas entre as ordenhas. A produção de leite é anotada em fichas individuais nas quais contêm anotações referentes à vida reprodutiva de cada animal. A partir das fichas de produção e reprodução foi editado um arquivo contendo o número da vaca, data de nascimento e parto, produção de leite diária e a data do controle leiteiro. Foram eliminadas as lactações que iniciaram o controle de produção 35 dias após o parto e lactações com menos de cinco controles, de modo a ter lactações com no mínimo 140 dias de duração. Os dados foram classificados em 12 estágios, de acordo com a periodicidade dos controles.

Seis funções matemáticas foram utilizadas para ajustar as lactações, tanto para a curva média como para as lactações individuais. $\mathrm{O}$ ajuste para a curva média foi realizado usando-se a média diária de produção de leite em cada estágio da lactação e usando-se todas as produções em cada estágio. Para as lactações individuais, o ajuste foi realizado considerando a produção de leite em dias.

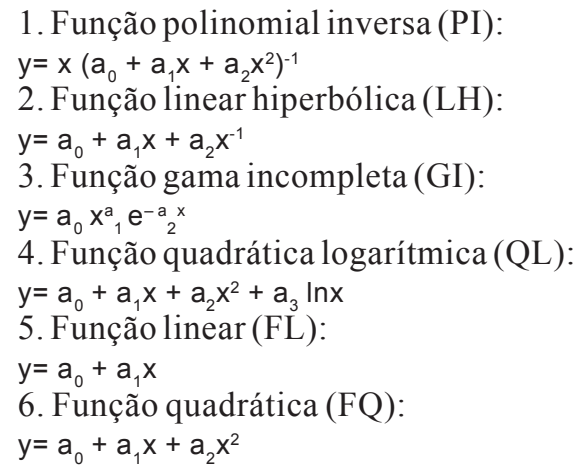

Para estas funções, y é a produção de leite $(\mathrm{kg})$; $a_{0}, a_{1}, a_{2}, e a_{3}$ são parâmetros da curva e serem estimados e $\mathrm{x}$ é o estágio da lactação.

Os parâmetros das funções foram estimados através de técnicas não lineares, usando-se o método modificado de GaussNewton disponível no PROC NLIN (SAS, 1996).

Os procedimentos estatísticos usados para escolha da melhor função foram:

a) Coeficiente de determinação ajustado

b) Gráfico de distribuição dos resíduos

c) Desvios entre as produções de leite totais observadas (PL) e estimadas (PLE): A produção total observada foi obtida pelo método escada corrigido (Bianchini Sobrinho, 1988).

As produções totais estimadas pelas funções foram obtidas pela somatória das produções estimadas em cada dia de lactação. Os desvios são dados por:

Desvio $(\%)=(P L-P L E) \times 100 / P L$

d) Estimativas de tempo de pico (TP), produção no pico (PP) e persistência de lactação (S) - As funções dos parâmetros como tempo de pico (TP), produção no pico (PP) e persistência de lactação (S) foram estimadas de acordo com cada modelo ajustado: 
1. Função polinomial inversa (Nelder, 1966);

$\mathrm{TP}=\sqrt{a_{0} / a_{2}} ; \mathrm{PP}=\left(2 \sqrt{a_{0} a_{2}}+\mathrm{a}_{1}\right)^{-1}$

2. Função linear hiperbólica (Bianchini Sobrinho, 1984);

$\mathrm{TP}=\sqrt{a_{1} / a_{2}} ; \mathrm{PP}=\mathrm{a}_{0}+2 \sqrt{a_{1} a_{2}}$ 1967);

3. Função gama incompleta (Wood,

$\mathrm{TP}=\frac{a_{1}}{a_{2}} ; \mathrm{PP}=\mathrm{a}_{0}\left(\frac{a_{1}}{a_{2}}\right)_{1}{ }_{1} \mathrm{e}_{1}^{-\mathrm{a}} ; \mathrm{S}=-\left(\mathrm{a}_{1}+1\right) \ln \mathrm{a}_{2} ;$

4. Função quadrática logarítmica (Bianchini Sobrinho, 1984);

$\mathrm{TP}=\frac{-a_{1} \pm \sqrt{a_{1}^{2}-8 a_{2} a_{3}}}{4 a_{2}} ; \mathrm{PP}=\mathrm{a}_{0}+\mathrm{a}_{1}(\mathrm{TP})+\mathrm{a}_{2}(\mathrm{TP})^{2}$ $+\mathrm{a}_{3} \ln (\mathrm{TP})$

5. Função quadrática;

$\mathrm{TP}=\frac{-a_{1}}{2 a_{2}} ; \mathrm{PP}=\mathrm{a}_{0}+\mathrm{a}_{1}(\mathrm{TP})+\mathrm{a}_{2}(\mathrm{TP})^{2}$

\section{RESULTADOSEDISCUSSÃO}

A média de produção de leite por lactação para raça Guzerá, em um período médio de 275 dias, foi de $2438 \mathrm{~kg}$, com coeficiente de variação de 25,86\%. Para vacas Sindi, a produção média foi de $2346 \mathrm{~kg}$, com coeficiente de variação de $27,29 \%$. Cobuci et al. (2000), estudando lactação de vacas Guzerá, observaram valores semelhantes aos obtidos no presente estudo. As curvas médias de lactação do rebanho estudado estão representadas na figura 1. De forma geral, o formato da curva apresentou tendência linear, com pico de produção no início da lactação, e tendência de queda contínua na produção diária. Formato semelhante foi obtido por Cobuci et al. (2000) para vacas Guzerá, Bianchini Sobrinho (1984) para vacas da raça Gir e El Faro (1996) para vacas Caracu.

Constam na tabela I os valores dos coeficientes de determinação ajustados para a curva média para as diferentes funções uti- lizadas. Observa-se que estes valores foram elevados, superiores a 0,93 para todas as funções, tanto para a raça Guzerá como para a raça Sindi, indicando boas aproximações e que para a estimativa da curva média todas as funções testadas poderiam ser utilizadas. Apesar desta tendência verificada para a curva média, para as lactações individuais nem sempre foram obtidos ajustes satisfatórios.

$\mathrm{Na}$ tabela II são apresentados às equações médias estimadas, as estimativas dos parâmetros, os componentes da curva de lactação e a quantidade de curvas típicas para cada função por raça. Os percentuais de curvas típicas obtidas foram inferiores aos obtidos por Cobuci et al. (2000) e El Faro e Albuquerque (2002) para as funções gama incompleta e linear hiperbólica e superiores para a função polinomial inversa. Em estudos de curva de lactação é importante quantificar o percentual de curvas típicas obtidos pelas funções, pois curvas atípicas são caracterizadas por apresentarem estimativas irreais e fora dos limites biológicos aceitáveis para os parâmetros da curva de lactação.

A função polinomial inversa (PI) ajustou

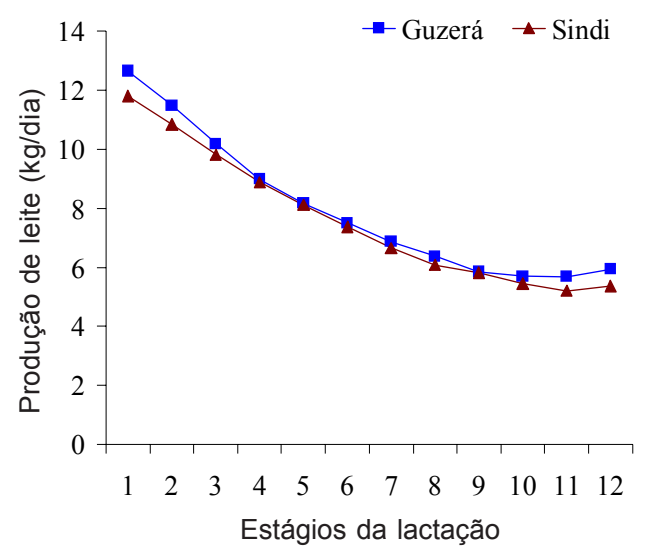

Figura 1. Curvas de lactação médias observadas para vacas Guzerá e Sindi. (Mean lactation curves observed for Guzerá and Sindi cows). 
Tabela I. Médias dos coeficientes de ajuste para os diferentes modelos utilizados para as raças estudadas. (Fit coefficient means for the different models used for the breeds studied).

\begin{tabular}{lcccccc}
\hline Raça & PI & LH & GI & QL & FL & FQ \\
\hline Guzerá & 0,941168 & 0,939491 & 0,940742 & 0,941057 & 0,937131 & 0,941335 \\
Sindi & 0,944371 & 0,943371 & 0,944193 & 0,944096 & 0,942381 & 0,944433 \\
\hline
\end{tabular}

bem (figuras 2 e 3 ) as lactações individuais, tanto para vacas Guzerá como para vacas Sindi, pois $61,4 \%$ e $57,2 \%$ das lactações estimaram $\mathrm{R}^{2}>0,80$ (tabela III). Estes valores foram superiores aos obtidos por Bianchini Sobrinho (1984), Pineda (1987) e El Faro e Albuquerque (2002). O R ${ }^{2}$ indica quanto da variação total foi explicada pelo modelo de regressão. Os valores dos parâmetros estimados pela função polinomial inversa indicam que houve grande variação destes parâmetros estimados para lactações individuais, principalmente para o parâmetro $\mathrm{a}_{0}$, em que o CV foi de $201,54 \%$. Valor de CV inferior $(83,43 \%)$ foi obtido por Pineda (1987) e valor superior $(479,4 \%)$ foi obtido por El Faro e Albuquerque (2002).

Para a PI, os valores de $\mathrm{a}_{0}$ devem ser positivos e próximos à zero. Neste estudo, algumas lactações, tanto de vacas Guzerá como Sindi, estimaram para esses parâmetros

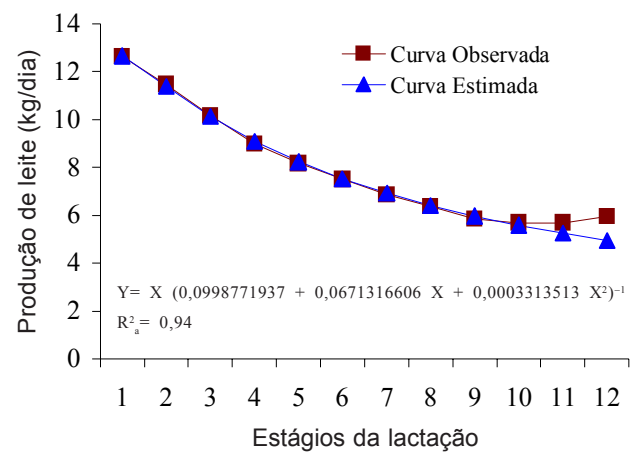

Figura 2. Curva de lactação média observada e estimada pela PI para vacas Guzerá. (Mean lactation curve, both observed and as estimated by the PI for Guzerá cows). valores irreais, gerando curvas atípicas. Para esta função, 76,59\% e 73,96\% das lactações de vacas Guzerá e Sindi, respectivamente, estimaram valores positivos para os parâmetros $a_{0}, a_{1}$ e $a_{2}$ (tabela II), condição necessária para que as curvas estimadas sejam consideradas típicas. Cobuci et al. (2000) estimaram, para vacas Guzerá, 60,3\% de curvas típicas utilizando a função polinomial inversa.

As estimativas das funções dos parâmetros TP e PP são apresentadas na tabela II. Para a PI o tempo de pico variou entre 4,98 dias a 68,01 dias com valor médio de 28,32 dias para a raça Guzerá e 4,49 dias a 77,23 dias com valor médio de 28,89 dias para Sindi, com respectivos CV de $35,87 \%$ e $77,23 \%$. A maioria das lactações típicas apresentou TP na segunda semana de lactação tanto para vacas Guzerá e como para vacas Sindi. Estes valores são inferio-

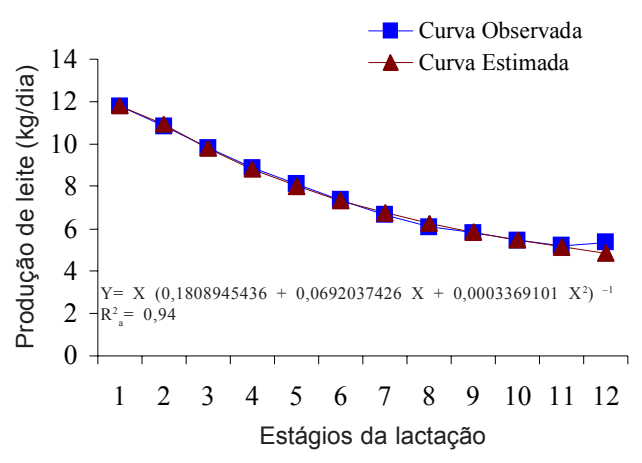

Figura 3. Curva de lactação média observada e estimada pela PI para vacas Sindi. (Mean lactation curve, both observed and as estimated by the PI for Sindi cows). 


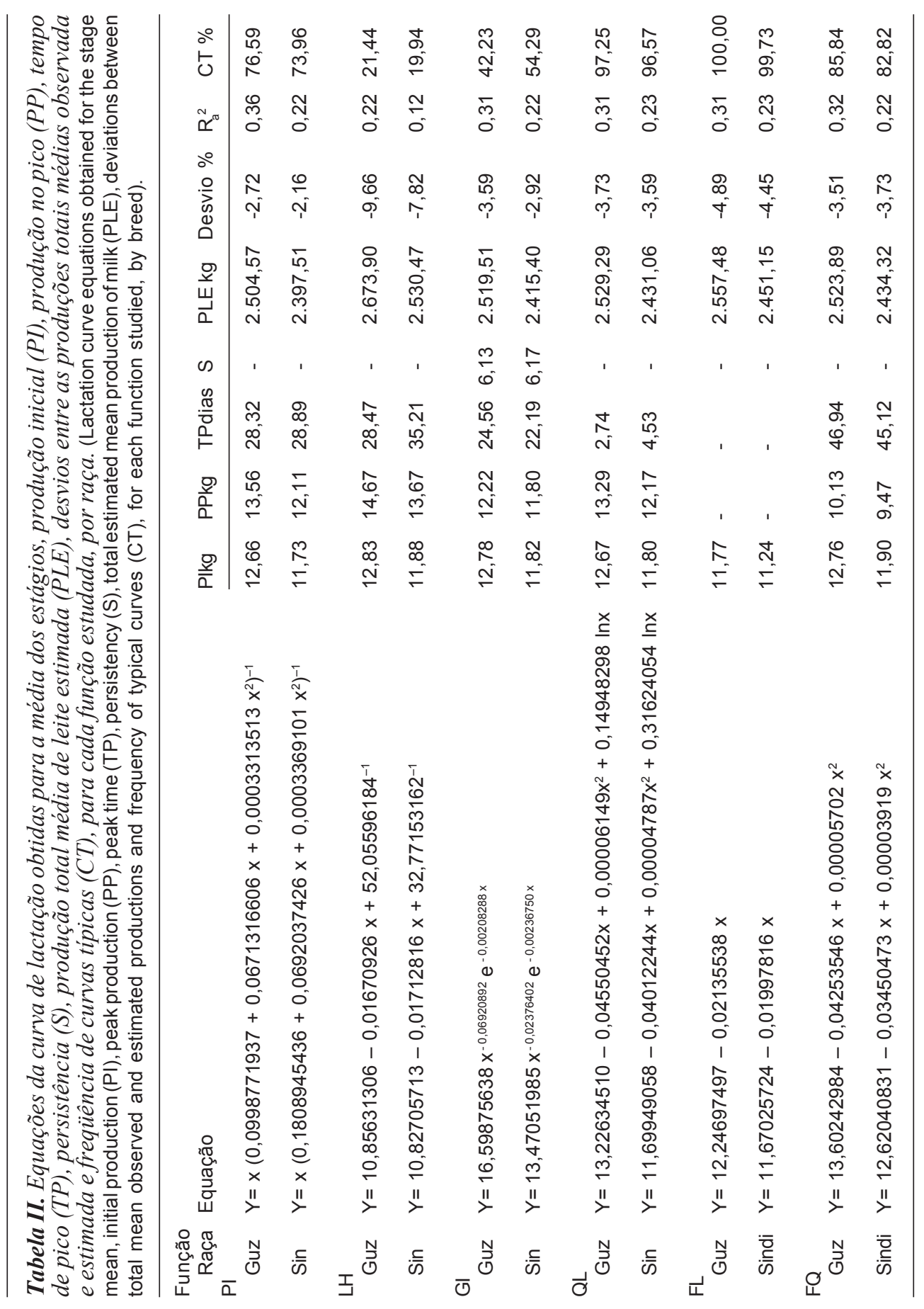

Archivos de zootecnia vol. 58, núm. 224, p. 700. 
Tabela III. Freqüência (\%) de lactações de vacas Guzerá (Guz) e Sindi (Sin) ajustadas pelas funções polinomial inversa (PI), linear hiperbólica (LH), gama incompleta (GI), quadrática logaritmica $(Q L)$, linear $(F L)$ e quadrática $(F Q)$ de acordo com os valores de $R^{2}$. (Frequency (\%) of lactations of Guzerá (Guz) and Sindi ( $\mathrm{Sin}$ ) cows fitted by the polynomial inverse (PI), hyperbolic lineal (LH), gamma incomplete $(\mathrm{GI})$, quadratic logarithmic $(\mathrm{QL})$, lineal $(\mathrm{FL})$ and quadratic $(F Q)$ functions according to the values of $\mathrm{R}_{\mathrm{a}}{ }^{2}$ ).

\begin{tabular}{lccccccccccccc}
\hline & \multicolumn{2}{c}{ PI } & \multicolumn{2}{c}{ LH } & \multicolumn{2}{c}{ Gl } & \multicolumn{2}{c}{ QL } & \multicolumn{2}{c}{ FL } & \multicolumn{2}{c}{$F Q$} \\
Valores $\mathrm{R}_{\mathrm{a}}{ }^{2}$ & Guz & Sin & Guz & Sin & Guz & Sin & Guz & Sin & Guz & Sin & Guz & Sin \\
\hline $\mathrm{R}_{\mathrm{a}}{ }^{2}>0,50$ & 98,4 & 91,7 & 95,5 & 78,7 & 97,4 & 88,5 & 95,4 & 92,8 & 94,4 & 92,6 & 94,8 & 93,2 \\
$\mathrm{R}_{\mathrm{a}}{ }^{2}>0,60$ & 94,2 & 84,1 & 93,9 & 74,5 & 95,7 & 81,2 & 90,4 & 86,7 & 89,2 & 86,2 & 89,7 & 86,9 \\
$\mathrm{R}_{\mathrm{a}}{ }^{2}>0,70$ & 79,9 & 73,1 & 74,2 & 66,0 & 79,5 & 70,8 & 73,7 & 72,9 & 74,3 & 71,8 & 73,4 & 72,7 \\
$\mathrm{R}_{\mathrm{a}}{ }^{2}>0,80$ & 61,4 & 57,2 & 56,1 & 42,6 & 66,7 & 50,0 & 55,0 & 54,2 & 55,0 & 53,7 & 54,0 & 54,5 \\
$\mathrm{R}_{\mathrm{a}}{ }^{2}>0,90$ & 39,2 & 37,9 & 37,9 & 19,1 & 47,9 & 29,2 & 29,6 & 32,5 & 31,2 & 31,9 & 29,4 & 31,2 \\
\hline
\end{tabular}

res aos obtidos por Pineda (1987), que estimou para esta função TP de 32,2 com CV de $100,82 \%$ e semelhantes aos obtidos por Bianchini Sobrinho (1984), que estimou TP de 10,76 dias, para vacas Gir em segunda lactação. A produção de leite no pico $(\mathrm{kg})$ foi de $13,56 \mathrm{~kg} /$ dia para vacas Guzerá e 12,11 $\mathrm{kg} /$ dia para vacas Sindi, o que gerou CV de $18,28 \%$ e $17,27 \%$, respectivamente. Estes valores são próximos à produção média de leite no início da lactação.

Os desvios médios entre as produções totais observadas e estimadas pela PI (tabela II) foram de $-2,72 \%$ para vacas Guzerá e $2,16 \%$ para vacas Sindi. Estes valores podem ser considerados baixos e ocorram, provavelmente, por ter-se utilizado dados de controle mensal, os quais são sujeitos a vícios.

Observando as figuras $\mathbf{4}$ e $\mathbf{5}$ nota-se que a PI representou bem a curva média do

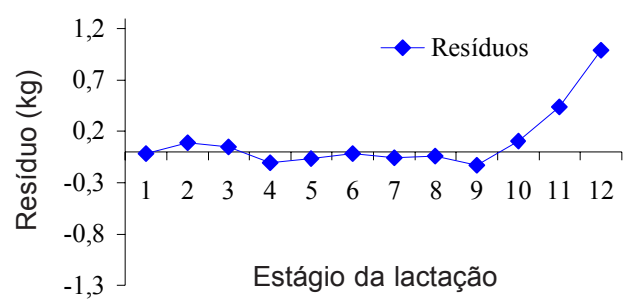

Figura 4. Distribuição dos resíduos estimados pela PI para vacas Guzerá. (Distribution of the residuals estimated by the PI for Guzerá cows). rebanho até o décimo estágio da lactação para animais Guzerá e até o décimo primeiro estágio para animais da raça Sindi. Para as duas raças, entre o décimo primeiro e o décimo segundo estágio houve uma nítida subestimação, porém com resíduos pequenos, menores que $1,0 \mathrm{~kg}$. Isto é verificado observando o gráfico de distribuição dos resíduos. Essa subestimação deve ter ocorrido devido ao fato de neste período o número de lactações ser bem menor em relação aos outros estágios.

A função linear hiperbólica não ajustou bem a curva de lactação de vacas Guzerá e Sindi, apesar de ter apresentado coeficiente de determinação ajustado elevado $\left(\mathrm{R}^{2}>0,90\right)$ para as curvas médias. Esta função não ajustou bem as lactações individuais do rebanho, pois estimaram, respectivamente, $56,1 \%$ e $42,6 \%$ de $\mathrm{R}_{\text {a }}^{2}>0,80$ para vacas Guzerá

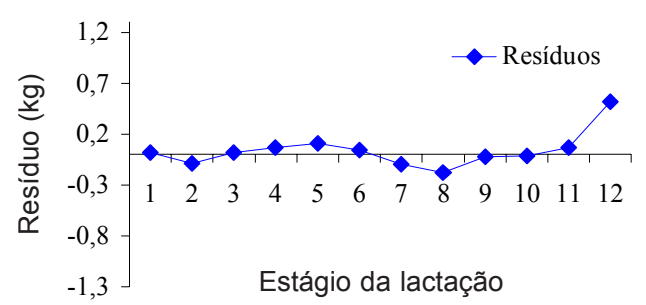

Figura 5. Distribuição dos resíduos estimados pela PI para vacas Sindi. (Distribution of estimated residuals by the PI for Sindi cows). 


\section{CRUZ, RIBEIRO E PIMENTA FILHO}

e Sindi, valores inferiores aos estimados pela PI. Além disto, a quantidade de curvas típicas foi muito baixa, $21,44 \%$ e $19,94 \%$, indicando ajustes pobres. Utilizando esta mesma função, El Faro e Albuquerque (2002) estimaram 52,32\% de curvas típicas.

Os valores estimados pela função linear hiperbólica (LH) para TP variaram de 1,96 a 69,07 dias com valor médio de 28,47 dias para raça Guzerá e de 6,57 a 76,98 dias com valor médio de 35,21 dias para raça Sindi e respectivos CV de 46,69\% e 49,10\%. Há uma grande variação para o TP, mostrando que há uma tendência do modelo estimá-lo no início da lactação. Neste estudo o tempo máximo para se alcançar o pico foi de 10 semanas para vaca Guzerá e 11 semanas para vacas Sindi. Estes valores estão dentro dos limites biológicos aceitáveis. Segundo El Faro e Albuquerque (2002), para raças taurinas o TP ocorre no máximo no terceiro mês de lactação. Bianchini Sobrinho (1984) encontrou valores para TP variando entre $14,17$ e 38,24 dias. A PP ( $\mathrm{kg})$ foi de $14,67 \mathrm{~kg} /$ dia para vacas Guzerá e 13,67 kg/dia para vacas Sindi. Os desvios médios entre as produções totais observadas e estimadas foram de $-9,66 \%$ para vacas Guzerá e -7,82\% para vacas Sindi. Quando se utilizou a produção total das lactações individuais esses desvios foram de $-17,26 \%$ e $14,44 \%$. Isto demonstra que esta função apresentou fraca estimativa para PLE.

As médias do parâmetro a estimado pela LH para as lactações individuais de vacas Guzerá variaram entre 3,61 e 20,19 e de 4,42 e 22,21 para Sindi. Este parâmetro está relacionado com a produção inicial. Assim, valores negativos são responsáveis por formas atípicas de curvas de lactação, indicando produção inicial inferior à zero. As estimativas dos parâmetros $\mathrm{a}_{1}$ e $\mathrm{a}_{2}$ deveriam ser negativas para estimarem picos de lactação. Para estes parâmetros foram encontrados altos coeficientes de variação, principalmente para o parâmetro $\mathrm{a}_{2}$, isto devido à ocorrência de valores mínimo e máximo extremos estimados para ele. Para
LH apenas 97 lactações de Guzerá e 72 lactações de Sindi estimaram tempo de pico e produção no pico, cujas estimativas dos parâmetros $a_{1}$ e $a_{2}$ foram negativas.

Os coeficientes de determinação ajustados pela função gama incompleta, para lactações individuais, demonstram que esta função proporcionou bom ajuste (tabela III). $\mathrm{R}^{2}>0,80$ foram obtidos por $66,7 \% \mathrm{e}$ $50,0 \%$ das lactações de vacas Guzerá e Sindi, respectivamente.

De acordo com as médias dos parâmetros estimados pela função gama incompleta(GI), observa-se que esta função apresentou problemas quando as estimativas destes, principalmente para o parâmetro $\mathrm{a}_{1}$, o qual apresentou elevados CV. Curvas típicas ocorrem quando todos os parâmetros são positivos. Segundo as restrições deste modelo o parâmetro $a_{1}$ deve variar de 0 a 1 e está associado com a produção até o pico de produção. O Parâmetro a está associado com a taxa de declínio após o pico. Para vacas Guzerá o parâmetro a variou de $-0,76$ a 0,44 com valor médio negativo $(-0,020)$. Para vacas Sindi, este parâmetro variou de $-0,38$ a 0,67 com valor médio de 0,015 . Este fato demonstra falhas nas estimativas, que foram responsáveis pela ocorrência de curvas atípicas.

O tempo de pico estimado pela função gama incompleta variou de 0,013 a 83,268 dias para vacas Guzerá, com média de 24,56 dias e CV de 65,74\%. Para vacas Sindi esta variação foi de 0,16 a 91,51 dias com média de 22,19 dias e CV de 89,24\%. Também verificou-se grande variação para o TP, pois os valores mínimos próximos à zero, indicam tendência de estimativas deste parâmetro logo no início da lactação ou até mesmo antes do parto. Essa mesma tendência foi observada por Pineda (1987) e El Faro e Albuquerque (2002). Arce e Correa (2005), estudando a lactação de vacas mestiças no México utilizando a GI encontraram valores médios para tempo de pico variando entre 51,27 e 62,81 dias, valores superiores aos verificados neste estudo. A produção no 
pico estimada pela FGI foi de $12,23 \mathrm{~kg}$ com CV de $19,23 \%$ para vacas Guzerá e $11,80 \mathrm{~kg}$ com CV de 18,65\% para vacas Sindi. Estes valores são próximos aos obtidos por Arce e Correa (2005) que estimaram PP valores entre 9,69 kg e 12,80 kg. Bianchini Sobrinho estimou PP de vacas da raça Gir através da FGI variando entre $8,40 \mathrm{~kg}$ e $15,91 \mathrm{~kg}$. A persistência(S) foi estimada em 6,13 com CV de $6,10 \%$ para vacas Guzerá e 6,17 com CV de $7,3 \%$. Este parâmetro da curva de lactação foi o que apresentou menor variação. Este fato foi também observado por El Faro e Albuquerque (2002) e Arce e Correa (2005). A persistência estimada para vacas Guzerá e Sindi foram praticamente iguais. Este fato deve-se pelo fato dos níveis de produção inicial serem muito próximos.

Os $\mathrm{R}^{2}$ estimados para as lactações individuais pela função quadrática logarítmica (QL) foram próximos aos estimados pelas funções linear e quadrática (tabela III) e semelhantes aos estimados por Bianchini Sobrinho(1984) e Pineda (1987). Esta função apresentou grande variação nas estimativas dos seus parâmetros. $\mathrm{O}$ parâmetro $\mathrm{a}_{0}$, relacionado às produções iniciais, foi o que apresentou menor variação. Estimativas negativas para este parâmetro indicam ocorrência de curvas atípicas. A QL estimou alta percentagem de curvas típicas, 97,25 e $96,57 \%$ para vacas Guzerá e Sindi, respectivamente.

As estimativas de TP e PP (tabela II) foram realizadas apenas para curva média. $\mathrm{O}$ TP estimado pela QL foi de 2,74 dias e 4,53 dias, indicando picos logo no início da lactação. A PP foi feita apenas considerando a curva média, já que este parâmetro é estimado em função do TP. Estes valores foram de $13,29 \mathrm{~kg}$ e $12,17 \mathrm{~kg}$ para vacas Guzerá e Sindi, respectivamente. A dificuldade encontrada para estimar os parâmetros TP e PP pela função quadrática logarítmica, para as lactações individuais, indica que ocorreram grandes variações nas formas das curvas de lactação o que é constatado pelos altos valores de CV para os parâmetros do modelo matemático.

A função linear é representada por dois parâmetros possuindo a vantagem de ser estimada através de regressão simples. O parâmetro $\mathrm{a}_{0}$ representa o intercepto e $\mathrm{a}_{1}$ à taxa de declínio médio da produção devendo ser negativo. Caso contrário à produção cresceria com o tempo e a curva seria uma reta ascendente. Devido a sua linearidade, a função linear não estima pico de produção, não detectando qualquer oscilação da produção. Esta função é utilizada para o ajuste de curvas de lactação de vacas zebuínas, devido sua característica linear (ElFaro, 1996).

Para raça Sindi a função linear estimou valores de $a_{1}$ positivos, sugerindo a ocorrência de curvas irreais. Esta função ajustou bem a curva média dos animais estudados. Para lactações individuais, o ajuste proporcionado pela FL foi semelhante as proporcionados pelas FQ e QL. A FL produziu fraca estimativa para PLE (tabela II). Todos os modelos superestimaram este parâmetro. A LH e FL foram os modelos que apresentaram as piores estimativas para PLE.

Para a função quadrática o parâmetro $\mathrm{a}_{0}$ é o intercepto, representando a produção inicial. Os parâmetros $a_{1}$ e $a_{2}$ são os coeficientes de regressão linear e quadrático. $\mathrm{O}$ parâmetro $\mathrm{a}_{2}$, em média foi positivo, e próximo à zero impossibilitando a estimativa de picos de produção. Para as lactações individuais, apenas nove lactações de vacas Guzerá estimaram TP e PP. Para vacas Sindi, apenas 17 lactações estimaram TP e PP.

Para vacas Guzerá o TP estimado pela FQ foi de 46,96 dias com CV de 55,25\%. Para vacas Sindi esses valores foram de 45,12 dias com CV de $80,76 \%$. A PP foi $10,13 \mathrm{~kg}$ e 9,47 kg para vacas Guzerá e Sindi.

\section{CONCLUSÕES}

De modo geral observou-se que a função polinomial inversa e a função gama incompleta proporcionaram melhores ajustes aos 


\section{CRUZ, RIBEIRO E PIMENTA FILHO}

parâmetros da curva média e individual. Estas funções também proporcionaram melhores estimativas dos componentes da

\section{BIBLIOGRAFIA}

Afifi, A.A. and V. Clark. 1984. Computer aided multivariate analysis. Lifetime Learning Publication. Belmont. California. 458 pp.

Arce, M.M.O. y J.C.S Correa. 2005. Factores que afectan la curva de lactancia de vacas Bos taurus $x$ Bos indicus en un sistema de doble propósito en el trópico húmedo de Tabasco, México. Téc. Pecu. Méx., 43: 127-137.

Bianchini Sobrinho, E. 1984. Estudo da curva de lactação em vacas da raça Gir. Tese (Doutorado em Genética). Faculdade de Medicina Veterinária de Ribeirão Preto. Universidade de São Paulo.Ribeirão Preto. 88 pp.

Bianchini Sobrinho, E. 1988. Estimativa de produção total de leite de vacas da raça Gir, baseada em controles semanais, quinzenais, mensais e bimestrais, obtenção de fatores multiplicativos. Tese (Livre Docência). Faculdade de Ciências Agrárias e Veterinárias. Universidade Estadual Paulista. Jaboticabal. 90 pp.

Brody, S., A.C. Ragsdale and C.W. Turner. 1923. The rate of decline of milk secretion with advance of the period of lactation. J. Gen. Physiol., 5: 441-444.

Cobucci, J.A., R.F. Euclydes, R.S. Verneque, R.L. Teodoro, P.S. Lopes e M.A. Silva. 2000. Curva de lactação na raça Guzerá. Rev. Bras. Zootecn., 29: 1332-1339.

Cobucci, J.A., R.F. Euclydes, R.L. Teodoro, R.S. Verneque, P.S. Lopes e M.A. Silva. 2001. Aspectos genéticos e ambientais da curva de lactação da raça guzerá. Rev. Bras. Zootecn., 30: 1204-1211.

Coby, J.M. and L.P. Le Du. 1978. On fitting curves to lactation data. Anim. Prod., 23: 127-133. curva de lactação de vacas Guzerá e Sindi, exploradas na região semi-árida do estado da Paraíba.

Cunha Filho, M. 2002. Curvas de lactação e de gordura em vacas da raça Sindi, no estado da Paraíba. Dissertação (Mestrado em Biometria). Departamento de Física e Matemática. Universidade federal Rural de Pernambuco. Recife. 57 pp.

El Faro, L. 1996. Estudo da curva de lactação de um rebanho da raça Caracu. Tese (Doutorado em Zootecnia). Faculdade de Ciências Agrárias e Veterinárias. Universidade Estadual Paulista. Jaboticabal. 172 pp.

El Faro, L. e L.G. Albuquerque. 2002. Comparação de alguns modelos matemáticos para o ajuste às curvas de lactação individuais de vacas da raça Caracu. Arq. Bras. Méd. Vet. Zootec., 54 295-302.

IBGE. 1990. Enciclopédia dos municípios brasileiros. Instituto de Geografia e Estatística. João Pessoa. PB. v. XVII.

Nelder, J.A. 1966. Inverse polynomials a useful group of multifactor response functions. Biometrics, 22: 128-141.

Pineda, A.M.G. 1987. Mathematical functions applied to dairy cow lactation curves. Thesis (Master of Science). University of Florida. Gainesville. 243 pp.

Ribeiro, M.N. 1997. Estudo da curva de lactação de um rebanho caprino no estado da Paraíba. Tese (Doutorado em Zootecnia). Faculdade de Ciências Agrárias e Veterinárias. Universidade Estadual Paulista. Jaboticabal. 91 pp.

SAS. 1996. Institute Inc. SAS/STAT User's guide. Version 6.12. SAS Institute Inc. Cary, NC. USA.

Wood, P.D.P. 1967. Algebraic model of the lactation curve on cattle. Nature, 216: 164-165

Archivos de zootecnia vol. 58, núm. 224, p. 704. 